\title{
MAPS PRESERVING EQUIVALENCE BY PRODUCTS OF INVOLUTIONS
}

\author{
GORDANA RADIĆ
}

Abstract. Let $\mathscr{B}(\mathscr{X})$ be the algebra of bounded linear operators on a complex Banach space $\mathscr{X}$. Two operators $A$ and $B \in \mathscr{B}(\mathscr{X})$ are said to be equivalent by products of involutions, if $A=T B S$ for $T$ and $S$ being a products of finitely many involutions. We will give description of linear bijective maps $\phi$ on $\mathscr{B}(\mathscr{X})$ satisfying that $\phi(A)$ and $\phi(B)$ are equivalent (i.e. $A=T B S$ for some invertible $T, S \in \mathscr{B}(\mathscr{X})$ ) whenever $A$ and $B$ are equivalent by products of involutions.

Mathematics subject classification (2010): 47B49, 15A86.

Keywords and phrases: Linear preserver, involution, equivalence, equivalence by products of involutions.

\section{REFERENCES}

[1] S. A. ARgyros AND R. G. Haydon, A hereditarily indecomposable $\mathscr{L}_{\infty}$-space that solves the scalar-plus-compact problem, Acta Math., 206, 1, 2011, 1-54.

[2] H.-K. DU AND G.-X. JI, Similarity-invariant subspaces and similarity-preserving linear maps, Acta Math. Sinica (Engl. Ser.), 18, 3, 2002, 489-498.

[3] S. Du AND J. Hou, Similarity invariant real linear subspaces and similarity preserving additive maps, Linear Algebra Appl., 377, 2004, 141-153.

[4] F. HiAI, Similarity preserving linear maps on matrices, Linear Algebra Appl., 97, 1987, 127-139.

[5] R. HoRn, C. K. LI AND N. K. TSING, Linear operators preserving certain equivalence relations on matrices, SIAM J. Matrix Anal. Appl, 12, 2, 1991, 195-204.

[6] J. Hou AND X.-L. ZHANG, Additive maps preserving similarity of operators on Banach spaces, Acta Math. Sinica (Engl. Ser.), 22, 1, 2006, 179-186.

[7] G. Ji, Similarity-preserving linear maps on $\mathscr{B}(\mathscr{H})$, Linear Algebra Appl., 360, 2003, 249-257.

[8] B. KuZMA, Additive mappings decreasing rank one, Linear Algebra Appl., 348, 2002, 175-187.

[9] C.-K. LI, P. ŠEMRL AND N.-S. SZE, Maps preserving the nilpotency of products of operators, Linear Algebra Appl., 424, 2007, 202-239.

[10] M. H. LIM, A note on similarity preserving linear maps on matrices, Linear Algebra Appl., 190, 1993, 229-233.

[11] F. Lu And C. Peng, Similarity-preserving linear maps on $\mathscr{B}(\mathscr{X})$, Studia Math., 209, 1, 2012, 1-10.

[12] B. S. Mityagin AND I. S. ÉDEL's ShtEIn, Homotopy type of linear groups of two classes of Banach spaces, Funct Anal Appl, 4, 3, 1970, 221-231.

[13] T. PeteK, Linear mappings preserving similarity on $\mathscr{B}(\mathscr{H})$, Studia Math., 161, 2, 2004, 177-186.

[14] T. PETEK AND G. RADIĆ, Linear preservers of equivalence relations on infinite-dimensional spaces, Studia Math., 238, 2, 2017, 101-119.

[15] H. RADJAVI, The group generated by involutions, Proceeding of the Royal Irish Academy, 81A, 1, 1981, 9-12.

[16] P. ŠEMRL, Similarity preserving linear maps, J. Operator Theory, 60, 1, 2008, 71-83.

[17] S. Shelah And J. STEPrans, A Banach-space on which there are few operators, Proc. Am. Math. Soc, 104, 1, 1988, 101-105.

[18] A. R. Sourour, Invertibility preserving linear maps on $\mathscr{L}(\mathscr{X})$, Trans. Amer. Math. Soc., 348, 1, 1996, 13-30.

[19] Z. QIN AND F. LU, Involution similarity preserving linear maps, Studia Math., Published online: 23.4.2019. 
[20] A. Wilansky, Subalgebras of $\mathscr{B}(\mathscr{X})$, Prodeeding sof the Royal Irish Academy, 29, 2, 1971, 355 360. 